\title{
Eficácia dos corticoides orais no alívio dos sintomas da síndroma do canal cárpico: qual a evidência atual?
}

Nuno Teles Pinto, ${ }^{1}$ João Teles, ${ }^{1}$ Maria João Serra, ${ }^{1}$ Vânia Fernandes ${ }^{1}$

\section{RESUMO}

Introdução: A síndroma do túnel cárpico (STC) é causada pela compressão do nervo mediano no seu trajeto através do túnel cárpico e é a neuropatia mais frequente do membro superior.

Objetivo: Rever e analisar a evidência atual sobre a eficácia dos corticoides orais no alívio dos sintomas em pacientes com síndroma do túnel cárpico.

Material e métodos: Pesquisa de guidelines (GL), ensaios clínicos aleatorizados e controlados (ECA), revisões sistemáticas (RS) e meta-análises (MA), publicadas entre 2009 e 2019, nas bases de dados e sítios de medicina baseada na evidência da PubMed, da Cochrane Library, DARE, Bandolier, TRIP Database, BMJ Evidence-Based Medicine, National Guideline Clearinghouse, NICE, Canadian Medical Association Practice Guidelines Infobase e Primary Care Clinical Practice Guidelines, utilizando-se os descritores MeSH: 'carpal tunnel syndrome' e 'corticosteroids'. Foi aplicada a escala Strength of Recommendation Taxonomy (SORT), da American Academy of Family Physicians, para atribuição dos níveis de evidência (NE) e das forças de recomendação (FR).

Resultados: Dos 172 artigos devolvidos da pesquisa efetuada foram selecionados seis: um ECA, quatro RS e uma norma de orientação clínica (NOC). A eficácia dos corticoides orais a curto-médio prazo no alívio sintomático da STC é evidenciada por vários ECA e RS metodologicamente bem concebidos. Na maioria dos estudos a administração oral de $20 \mathrm{mg}$ de prednisolona por duas semanas conduz ao alívio temporário dos sintomas.

Conclusões: A corticoterapia oral pode ser usada no alívio temporário dos sintomas do STC a curto prazo, num regime de $20 \mathrm{mg}$ diários de prednisolona durante duas semanas (SORT A). O seu uso no tratamento a médio e a longo prazo apresenta um nível de evidência mais baixo (SORT B).

Palavras-chave: Síndroma do túnel cárpico; Corticosteroides.

\section{INTRODUÇÃO}

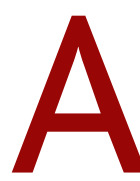
síndroma do túnel cárpico (STC) é causada pela compressão do nervo mediano no seu trajeto através do túnel cárpico. Ainda que não se conheça o mecanismo exato que causa esta patologia, pensa-se que está relacionado com o aumento da pressão no túnel cárpico, resultando numa compressão mecânica e isquemia local com consequente lesão do nervo mediano. ${ }^{1}$ Trata-se da neuropatia compressiva do membro superior e da patologia não

1. Médico Interno de Medicina Geral e Familiar. USF Fânzeres - ACeS de Gondomar. Fânzeres, Portugal. traumática da mão mais comum. ${ }^{2}$ Apresenta uma prevalência de 5,3\% nas mulheres e de 2,1\% nos homens na população em geral, sendo mais comum entre os 41 e os 60 anos..$^{3-4}$ Outros fatores de risco independentes são a obesidade, o hipotiroidismo, a diabetes mellitus, a artrite reumatoide, estados edematosos, os movimentos repetitivos do punho e a gravidez. ${ }^{5}$

Os sintomas da STC englobam sintomas sensitivosdor, parestesias e hipoestesias da região da mão inervada pelo nervo mediano, isto é, primeiro, segundo, terceiro dedos e porção radial do quarto dedo da mão - e sintomas motores - fraqueza e, em casos mais prolongados no tempo, atrofia dos músculos da região 
tenar. Habitualmente os sintomas agravam durante a noite, podendo levar a vários despertares noturnos e a má qualidade do sono. ${ }^{2,6} \mathrm{O}$ diagnóstico é clínico, podendo ser realizada a eletromiografia e, eventualmente, a ecografia ou ressonância magnética nuclear no caso de existirem dúvidas no diagnóstico. ${ }^{6}$

A STC tem um grande impacto económico para os serviços de saúde, relacionado com o tratamento desta patologia, e um forte impacto social, na medida em que é uma causa importante de absentismo laboral e de diminuição da qualidade de vida. ${ }^{6} \mathrm{O}$ médico de família é quase invariavelmente o primeiro profissional de saúde a quem os doentes recorrem por causa desta patologia, cabendo-lhe a responsabilidade do diagnóstico, tratamento sintomático e eventual referenciação para cuidados de saúde secundários com vista à melhor caracterização da doença ou tratamento cirúrgico, que geralmente passa pela descompressão cirúrgica do nervo mediano. Os anti-inflamatórios não esteroides (AINE) e os fármacos gabapentinoides (gabapentina e pregabalina) constituem as opções farmacológicas orais mais usadas no controlo sintomático, apesar da evidência ser escassa e de frequentemente não induzirem melhoria significativa. ${ }^{18}$ Os corticoides orais são usados de forma bastante esporádica como alternativa terapêutica, uma vez que a sua eficácia na STC não é clara ou pouco conhecida, ao passo que a infiltração local de corticoides colhe uma maior unanimidade ao nível da sua eficácia. Assim, evidencia-se a importância da identificação de opções terapêuticas com eficácia comprovada na STC, sendo estas fundamentais no controlo sintomático em doentes com diagnóstico recente, em que uma abordagem expectante é justificada, e também naqueles que recusam tratamento cirúrgico ou enquanto aguardam ser chamados para cirurgia. ${ }^{7}$

Desta forma, os autores propõem-se realizar uma revisão baseada na evidência (RBE), no sentido de rever e analisar a evidência atual sobre a eficácia dos corticoides orais no alívio dos sintomas em pacientes com STC.

\section{MÉTODOS}

Foi realizada uma pesquisa de guidelines (GL), ensaios clínicos aleatorizados e controlados (ECA), revisões sistemáticas (RS) e meta-análises (MA), nas bases de dados da PubMed, The Cochrane Library, DARE, Bandolier, TRIP Database, BMJ Evidence-Based Medici- ne, National Guideline Clearinghouse, NICE, Canadian Medical Association Practice Guidelines Infobase e Primary Care Clinical Practice Guidelines, publicados entre 11 de abril de 2009 e 11 de abril de 2019, em português, inglês, francês e espanhol. Foram utilizados os termos MeSH: 'carpal tunnel syndrome' e 'corticosteroids.' Foi aplicada a escala Strength of Recommendation Taxonomy (SORT), da American Academy of Family Physicians, para atribuição dos níveis de evidência (NE) e forças de recomendação (FR). Para avaliação da qualidade metodológica (risco de viés) do ECA incluído nesta revisão e dos ECA das revisões sistemáticas incluídas foram aplicados os 12 critérios de qualidade metodológica/fontes de risco de viés publicados nas guidelines de metodologia para revisões sistemáticas da Cochrane Back Review Group. ${ }^{19}$

Os critérios utilizados para a inclusão dos estudos nesta revisão foram definidos segundo o modelo PICOS (população, intervenção, comparação, outcome e tipo de estudo). Foi definida como população-alvo indivíduos adultos de ambos os sexos, com diagnóstico de STC e em que a intervenção terapêutica consistiu na administração de corticoterapia oral em comparação com outra intervenção terapêutica isolada ou ausência de qualquer tratamento. O outcome estudado foi a diminuição dos sintomas sensitivos e motores da STC. Foram excluídos artigos duplicados, artigos de opinião, revisões clássicas, sumários de sítios na Internet, discordância com o objetivo desta RBE, estudos em que a população incluísse crianças, grávidas, doentes já operados a STC ou com história de tratamento com infiltração local de corticoides nas últimas 12 semanas e doentes com causas secundárias de STC, como hipotiroidismo, artrite reumatoide ou diabetes mellitus.

A seleção dos artigos para revisão foi feita em duplicado por todos os autores de forma emparelhada e os conflitos na seleção dos mesmos foram resolvidos por concordância. A avaliação das FR e NE dos artigos incluídos foi também debatida por todos os autores.

\section{RESULTADOS}

Da pesquisa realizada resultaram 172 artigos, seis dos quais cumpriam os critérios de inclusão: uma NOC, um ECA e quatro RS (Figura 1). A unidade de análise definida pelos autores relativamente às RS são os estudos primários nos quais estas se baseiam. 


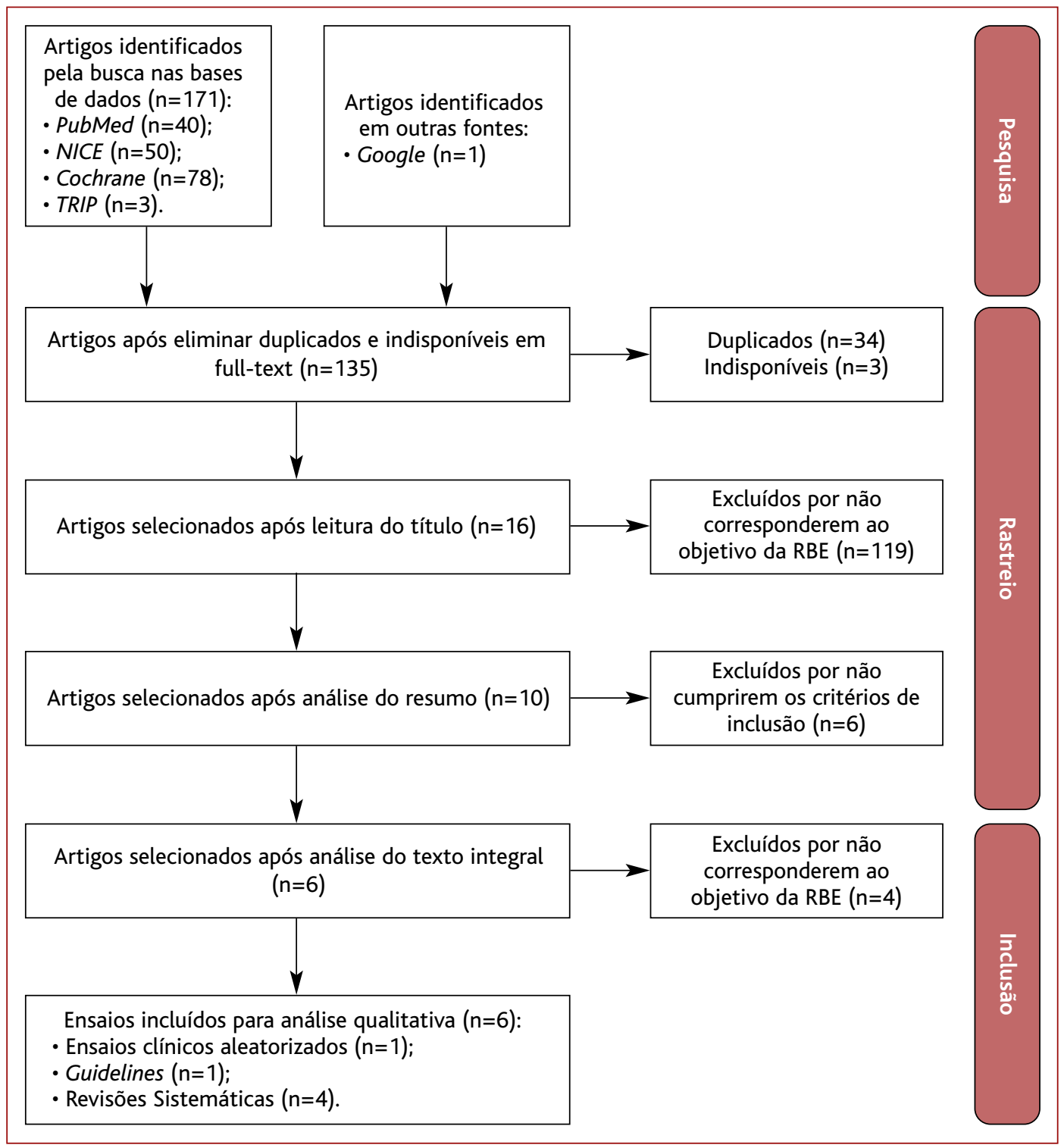

Figura 1. Processo de seleção dos artigos.

\section{Ensaios clínicos aleatorizados}

O único ECA que responde aos critérios de inclusão (PICOS) na pesquisa efetuada é um estudo realizado no Hospital Hasan Sadikin, na Indonésia, que teve como objetivo principal comparar a eficácia da infiltração local de triamcinolona com a triamcinolona oral no alívio sintomático da STC. ${ }^{5}$ Foram incluídos pacientes com STC idiopática sem atrofia da região tenar da(s) mão(s) afetada(s) e sem contraindicações para corticoterapia. Foram excluídos pacientes com patologias potencialmente causadoras de STC ou com contraindicações para a realização de corticoterapia, como presença de úlcera péptica, infeções agudas e infeção fúngica sistémica, e doentes que tenham feito corticoterapia oral ou injetável nos últimos três meses. A STC foi definida de acordo com os critérios de diagnóstico de Rempel. Estes critérios são a combinação dos sintomas (dor, parestesias ou hipoestesias) no território de distribuição do nervo mediano ou fraqueza muscular da região tenar, teste de Tinel ou de Phalen positivos e a 


\section{TABELA 1. Ensaios clínicos incluídos na revisão e suas características}

\begin{tabular}{|c|c|c|c|c|c|}
\hline Ensaio Clínico & Metodologia & Intervenção & Outcome & Conclusões & NE \\
\hline $\begin{array}{l}\text { Dewi et al. } \\
(2009)^{5}\end{array}$ & $\begin{array}{l}\text { Ensaio clínico } \\
\text { controlado e } \\
\text { aleatorizado. } \\
\text { Alocação randomizada } \\
\text { de } 50 \text { pacientes a dois } \\
\text { grupos de tratamentos } \\
\text { diferentes. }\end{array}$ & $\begin{array}{l}\text { Grupo 1. Tratados com } \\
\text { triamcinolona } 16 \mathrm{mg} \text { por } \\
\text { dia durante } 2 \text { semanas e } \\
\text { depois } 8 \mathrm{mg} \text { por dia } \\
\text { durante } 2 \text { semanas + } \\
\text { injeção de placebo. } \\
\text { Grupo } 2 \text {. Injeção de } \\
\text { triamcinolona + placebo } \\
\text { oral durante } 4 \text { semanas. } \\
\text { Todos os participantes } \\
\text { foram avaliados no início } \\
\text { e no fim do tratamento } \\
\text { com a global symptom } \\
\text { score e com testes de } \\
\text { função neurofisiológica. }\end{array}$ & $\begin{array}{l}\text { Global symptom score e } \\
\text { resultados dos testes de } \\
\text { avaliação } \\
\text { neurofisiológica. }\end{array}$ & $\begin{array}{l}\text { A corticoterapia local } \\
\text { infiltrada é mais eficaz } \\
\text { na melhoria sintomática } \\
\text { e nos parâmetros } \\
\text { neurofisiológicos do } \\
\text { que a corticoterapia } \\
\text { sistémica oral. }\end{array}$ & 1 \\
\hline
\end{tabular}

presença de anomalias detetadas nos estudos de condução nervosa. Foi feita uma alocação randomizada de 50 pacientes, selecionados de acordo com estes critérios, em dois grupos: um grupo foi tratado com triamcinolona oral $16 \mathrm{mg}$ por dia durante duas semanas, seguido de triamcinolona oral $8 \mathrm{mg}$ por dia durante mais duas semanas e uma injeção local de placebo. O outro grupo recebeu uma injeção de triamcinolona e placebo oral durante quatro semanas. Todos os participantes foram avaliados através do Global Symptom Score (GSS) e através de testes de função neurofisiológica. O GSS foi avaliado imediatamente antes do início da intervenção, às duas e às quatro semanas. Já os testes de função neurofisiológica foram avaliados apenas antes da intervenção terapêutica e quatro semanas após.

No grupo submetido a infiltração houve diminuição significativa no GSS ao fim da segunda e quarta semana ( $p<0,001$ e $p=0,007$, respetivamente). No grupo tratado com corticoide oral houve uma diminuição significativa ao fim da segunda semana $(p=0,003)$, mas não à quarta semana $(p=0,214)$. Não houve diferenças significativas na média do GSS às duas semanas entre ambos os grupos $(p=0,260)$. No grupo da infiltração constatou-se uma média no GSS às quatro semanas significativamente menor comparativamente ao grupo do tratamento oral $(p=0,026)$. A melhoria na latência motora distal às quatro semanas foi significativa em ambos os grupos, com o grupo do tratamento por infiltração a demonstrar melhorias mais significativas nos parâmetros neurofisiológicos do que o grupo submetido a corticoterapia oral (Tabela 1).

\section{Estudos primários que compõem as revisões sistemáticas}

Da pesquisa realizada foram incluídos os estudos primários de quatro RS (Tabela 2).

Hamamoto e colaboradores realizaram, em 2009, uma revisão sistemática da literatura sobre a eficácia de fármacos anti-inflamatórios esteroides e não-esteroides no tratamento de casos ligeiros a moderados de STC. ${ }^{7}$ Foram incluídos apenas ensaios clínicos aleatorizados, duplamente cegos. Seis publicações referentes a cinco ensaios foram incluídas na revisão. Destes, três ensaios incluíram a administração de corticoides orais.

No ensaio clínico randomizado e duplamente cego de Chang e colaboradores, de 2002, incluído nesta RS, 53 pacientes foram tratados com prednisolona oral por quatro semanas (duas semanas com 20mg/dia, seguido de duas semanas com 10mg/dia) e outros 56 pacientes foram tratados durante quatro semanas com o esquema de duas semanas de prednisolona $20 \mathrm{mg} / \mathrm{dia}$, seguido de duas semanas com placebo oral. ${ }^{8}$ Todos os 


\begin{tabular}{|c|c|c|c|}
\hline $\begin{array}{l}\text { Revisão } \\
\text { Sistemática }\end{array}$ & Objetivo da revisão e estudos incluídos & Conclusões & NE \\
\hline $\begin{array}{l}\text { Hamamoto } \\
\text { et al. (2009) }\end{array}$ & $\begin{array}{l}\text { Revisão sistemática da literatura sobre a } \\
\text { eficácia de fármacos anti-inflamatórios } \\
\text { esteroides e não-esteroides no tratamento de } \\
\text { casos ligeiros a moderados de síndroma do } \\
\text { túnel cárpico. Foram incluídos cinco ensaios } \\
\text { clínicos aleatorizados, duplamente cegos. }\end{array}$ & $\begin{array}{l}\text { Os corticoides (orais ou infiltrados localmente) } \\
\text { proporcionam alívio sintomático significativo a curto } \\
\text { prazo na síndroma do túnel cárpico, sendo que a } \\
\text { infiltração local de corticoides apresenta uma eficácia } \\
\text { superior em comparação com a corticoterapia oral. }\end{array}$ & 1 \\
\hline $\begin{array}{l}\text { Huisstede et al. } \\
(2010)^{2}\end{array}$ & $\begin{array}{l}\text { Revisão sistemática da literatura sobre a } \\
\text { eficácia de intervenções não cirúrgicas para o } \\
\text { tratamento da síndroma do túnel cárpico. } \\
\text { Foram selecionadas duas revisões } \\
\text { sistemáticas }{ }^{11,13} \text { e } 26 \text { ensaios clínicos } \\
\text { aleatorizados. }\end{array}$ & $\begin{array}{l}\text { Existe evidência forte de que o tratamento } \\
\text { sintomático da síndroma do túnel cárpico com } \\
\text { corticoides orais é eficaz a curto prazo e evidência } \\
\text { moderada dessa mesma eficácia a médio prazo. A } \\
\text { infiltração local de corticoides é superior aos } \\
\text { corticoides orais a curto e a médio prazo. No entanto, } \\
\text { os benefícios dos corticoides localmente injetados } \\
\text { não se mantêm a longo prazo, como acontece com os } \\
\text { corticoides orais. }\end{array}$ & 1 \\
\hline $\begin{array}{l}\text { Ono et al. } \\
(2010)^{6}\end{array}$ & $\begin{array}{l}\text { Revisão sistemática da literatura sobre a } \\
\text { eficácia das opções terapêuticas para a } \\
\text { síndroma do túnel cárpico. Incluiu a guideline } \\
\text { da American Academy of Orthopaedic Surgeons } \\
\text { (AAOS) e uma revisão sistemática. }{ }^{15}\end{array}$ & $\begin{array}{l}\text { Os corticoides, particularmente os que são infiltrados } \\
\text { localmente, são eficazes no alívio dos sintomas da } \\
\text { síndroma do túnel cárpico. Como tal, os corticoides } \\
\text { orais e infiltrados são úteis no tratamento inicial da } \\
\text { síndroma do túnel cárpico no que toca ao alívio dos } \\
\text { sintomas, no entanto, os seus efeitos são temporários. }\end{array}$ & 1 \\
\hline $\begin{array}{l}\text { Huisstede et al. } \\
(2018)^{2}\end{array}$ & $\begin{array}{l}\text { Revisão sistemática da literatura sobre a } \\
\text { eficácia da terapêutica oral e da corticoterapia } \\
\text { injetável na síndroma do túnel cárpico. Foram } \\
\text { incluídos dois ensaios clínicos aleatorizados de } \\
\text { alta qualidade e uma revisão sistemática de } \\
\text { alta qualidade. }\end{array}$ & $\begin{array}{l}\text { Existe forte evidência de que os corticoides orais são } \\
\text { eficazes no tratamento da síndroma do túnel cárpico } \\
\text { em comparação com o placebo, a curto prazo. } \\
\text { Existe evidência moderada de que os corticoides orais } \\
\text { são mais eficazes no tratamento da síndroma do } \\
\text { túnel cárpico em comparação com as ortóteses do } \\
\text { punho, a curto prazo. } \\
\text { Não existe evidência da eficácia dos corticoides orais } \\
\text { no tratamento da síndroma do túnel cárpico a longo } \\
\text { prazo. } \\
\text { Existe evidência forte de que os corticoides infiltrados } \\
\text { são mais eficazes do que os corticoides orais a curto } \\
\text { prazo no tratamento da síndroma do túnel cárpico. }\end{array}$ & 1 \\
\hline
\end{tabular}

participantes foram avaliados no início e no fim do tratamento com o GSS e com eletromiografia. Avaliações intercalares com o GSS foram realizadas aos três, seis, nove e doze meses. Ambos os grupos demonstraram uma diminuição significativa do GSS e melhoria significativa nos parâmetros neurofisiológicos na eletromiografia, mas não foram encontradas diferenças estatisti- camente significativas entre os mesmos, quer para o GSS quer para os parâmetros da eletromiografia.

No ensaio clínico randomizado e duplamente cego de Wong e colaboradores, de 2001, 30 pacientes foram tratados com infiltração local de metilprednisolona e 10 dias de placebo oral e 30 pacientes foram tratados com 25mg de prednisolona oral durante 10 dias 
e uma infiltração local de placebo. ${ }^{9}$ Foram avaliados os sintomas dos doentes através do GSS em ambos os grupos antes da intervenção e às duas, oito e doze semanas após a intervenção. Verificou-se uma diminuição estatisticamente significativa na média do GSS no grupo sujeito à injeção com metilprednisolona às duas, oito e doze semanas $(p<0,001)$ e no grupo sujeito a prednisolona oral às duas e oito semanas $(p=0,001)$, mas não às doze semanas $(p=0,03)$. Não houve diferenças estatisticamente significativas na média do GSS entre ambos os grupos antes da intervenção $(p=0,70)$ e duas semanas após $(p=0,07)$. Às oito e às doze semanas os pacientes tratados com injeção local de metilprednisolona apresentaram uma média do GSS significativamente menor comparativamente aos pacientes tratados com prednisolona oral ( $p=0,004$ e $p=0,002$, respetivamente).

Hui e colaboradores realizaram, em 2004, o follow-up destes doentes até 19 meses após o início da intervenção, através dum novo ensaio clínico randomizado duplamente cego. ${ }^{10}$ Os doentes permaneceram divididos nos mesmos dois grupos - o grupo que fora submetido a metilprednisolona injetável e o grupo que fora submetido a prednisolona oral no estudo efetuado por Wong e colaboradores. ${ }^{9} \mathrm{~A}$ todos os doentes sintomáticos no final do estudo de original ${ }^{9}$ foram prescritas ortóteses do punho de uso noturno, não tendo sido permitidas co-intervenções terapêuticas adicionais. Durante os 19 meses do período de follow-up, os doentes foram reavaliados clinicamente através do GSS, aproximadamente aos sete, 11, 15 e 19 meses após a intervenção, tendo sido este o outcome primário deste ECA. Em cada uma dessas avaliações foi também oferecida a opção terapêutica de descompressão cirúrgica aos doentes de ambos os grupos, tendo sido a proporção de doentes submetidos a cirurgia um outcome secundário. Não se verificaram diferenças estatisticamente significativas entre ambos os grupos no que se refere à média do GSS $(p>0,05)$ e no que respeita à proporção de doentes submetidos a cirurgia $(p>0,05)$ aos sete, 11 , 15 e aos 19 meses após a intervenção.

Desta forma, os autores desta RS concluíram que embora os estudos neurofisiológicos não tenham mostrado grandes diferenças resultantes das várias modalidades de utilização de corticoides, os corticoides (orais ou infiltrados localmente) proporcionam alívio sintomático significativo a curto prazo na STC, sendo que a infiltração local de corticoides apresenta uma eficácia superior em comparação com a corticoterapia oral.

Huisstede e colaboradores levaram a cabo, em 2010, uma revisão sistemática da literatura sobre a eficácia de intervenções não cirúrgicas para o tratamento da STC. ${ }^{2}$ Foram selecionados 26 ECA e duas RS. Uma das $\mathrm{RS}^{11}$ citou um ECA de alta qualidade realizado por Mishra e colaboradores em 2006, ${ }^{12}$ que comparou o tratamento de prednisolona oral $20 \mathrm{mg} /$ dia durante duas semanas seguidos de $10 \mathrm{mg} /$ dia por mais duas semanas, com o tratamento com ortótese do punho na STC. Foram incluídos 40 pacientes com STC, sendo que 20 foram aleatorizados para o grupo da terapêutica oral e os outros 20 para o grupo da ortótese do punho. O outcome primário foi a avaliação dos sintomas clínicos medidos pelo Functional Status Score (FSS) e pelo Symptom Severe Scale (SSS), enquanto os outcomes secundários foram a medição da latência distal sensitiva e motora do nervo mediano e a velocidade de condução. Os outcomes primários e os outcomes secundários foram avaliados em ambos os grupos antes da intervenção e três meses após. Decorridos três meses registou-se uma melhoria clínica estatisticamente significativa medida através do SSS e do FSS em ambos os grupos $(p<0,001)$. A latência distal sensitiva do nervo mediano e a velocidade de condução também melhoraram significativamente em ambos os grupos ao fim de três meses. Já a melhoria na latência distal motora foi estatisticamente significativa $(p=0,001)$ decorridos três meses no grupo submetido a prednisolona oral, mas não no grupo submetido a ortótese do punho $(p=0,139)$. Comparando os dois grupos, a melhoria clínica avaliada pelo FSS foi significativamente maior no grupo que fez prednisolona oral $(p=0,03)$. No entanto, não se verificaram diferenças significativas entre os grupos ao nível do SSS e ao nível da latência distal sensitiva e da velocidade de condução.

Foi incluída uma RS da Cochrane, que incluiu quatro ECA de alta qualidade que compararam a eficácia dos corticoides orais com o placebo, demonstrando melhorias clínicas significativas em favor dos corticoides às duas semanas de tratamento, com um dos ensaios a indicar a manutenção dessa melhoria clínica às quatro semanas. ${ }^{13}$ Este último foi conduzido por Chang e colaboradores, em 1998, e trata-se de um ECA prospetivo duplamente cego que comparou três diferentes 
terapêuticas orais no STC durante quatro semanas com o placebo. ${ }^{14}$ Um grupo foi tratado com o diurético triclormetiazida $2 \mathrm{mg} / \mathrm{dia}(n=16)$, outro grupo com o anti-inflamatório não esteroide tenoxicam-SR $20 \mathrm{mg}$ /dia $(n=18)$, outro grupo com prednisolona oral $20 \mathrm{mg} / \mathrm{dia}$ durante duas semanas seguido de duas semanas de $10 \mathrm{mg} /$ dia de prednisolona oral $(n=23)$ e o outro grupo foi tratado com placebo oral ( $n=16)$. O outcome primário foi a sintomatologia dos pacientes avaliados pelo GSS, que foi aplicado antes e depois da intervenção, no final da quarta semana. Não se verificaram diminuições significativas na média do GSS no grupo placebo e nos grupos tratados com o diurético e com o anti-inflamatório não esteroide. No entanto, verificou-se uma diminuição significativa na média do GSS no grupo tratado com corticoide oral. Esta revisão sistemática também incluiu o estudo de Chang et al, ${ }^{8}$ de Wong et al ${ }^{\beta} \mathrm{e}$ de Hui et $a l,{ }^{10}$ já citados na revisão de Hamamoto e colaboradores. ${ }^{7}$ Os autores da presente RS concluem que os corticoides orais têm evidência de eficácia a curto e a médio prazo, mas não a longo prazo. Os corticoides infiltrados localmente são superiores aos corticoides orais a curto e a médio prazo, mas o seu efeito não é sustentado a longo prazo.

Ono e colaboradores, em 2010, conduziram uma RS da literatura sobre a eficácia das opções terapêuticas para a STC. ${ }^{6}$ Foram pesquisadas RS, meta-análises, ECA e guidelines nas bases de dados da MEDLINE e da Cochrane Library. A seleção dos estudos, a extração dos dados e a avaliação metodológica foi realizada por dois revisores independentes. Esta revisão cita a guideline da American Academy of Orthopaedic Surgeons (AAOS) de 2007, que recomenda o tratamento da STC com infiltração local de corticoides antes de se considerar a terapêutica cirúrgica e com corticoides orais como alternativa aos corticoides infiltrados localmente; salienta ainda que os corticoides são mais eficazes no alívio dos sintomas da STC do que anti-inflamatórios não esteroides ou diuréticos, embora apresentem maior risco de efeitos adversos. Este estudo incluiu também uma RS, conduzida por Marshall e colaboradores, que concluiu que as injeções locais de corticoides são mais eficazes do que o tratamento com corticoides orais no alívio dos sintomas da STC. ${ }^{15}$ Estes autores concluíram que os corticoides, particularmente os que são infiltrados localmente, são eficazes no alívio dos sintomas da STC.
Como tal, os corticoides orais e infiltrados são úteis no tratamento inicial da STC no que toca ao alívio dos sintomas; no entanto, os seus efeitos são temporários.

Huisstede e colaboradores realizaram, em 2018, uma RS da literatura sobre a eficácia da terapêutica oral e da corticoterapia injetável na STC. ${ }^{2}$ Neste estudo foram incluídos os ECA de alta qualidade de Chang et al e de Hui et $a l^{10}$ e uma RS que incluiu o ECA de alta qualidade de Wong et al, ${ }^{9}$ já citados na RS de Hamamoto et al, de 2009. ${ }^{7}$ Adicionalmente, neste estudo também foram incluídos o ECA de alta qualidade de Mishra e colaboradores de $2006^{12}$ e uma RS da Cochrane,,$^{13}$ que incluiu quatro ECA de alta qualidade, já citados na revisão de Huisstede e colaboradores, de 2010. ${ }^{16}$

Estes autores concluíram que existe forte evidência para a eficácia dos corticoides orais versus placebo no alívio dos sintomas do STC a curto prazo. Nenhuma evidência foi encontrada para a eficácia dos corticoides orais a longo prazo. Estes autores concluíram também que existe evidência moderada de que os corticoides orais são mais eficazes no tratamento do STC em comparação com as ortóteses do punho a curto prazo. Além disso, foi também concluído que os corticoides infiltrados são mais eficazes do que os corticoides orais a curto prazo no tratamento da STC.

\section{Guidelines}

Da pesquisa efetuada apenas uma guideline cumpriu os critérios de inclusão (Tabela III). Trata-se da guideline da American Academy of Orthopaedic Surgeons, de 2016, sobre a abordagem terapêutica da STC. ${ }^{17}$ Esta guideline recomenda os corticoides orais na melhoria dos outcomes dos pacientes com STC em comparação com o placebo. Esta recomendação é de força moderada e é suportada nesta guideline por um nível de evidência moderado, baseado no ECA de alta qualidade de Chang e colaboradores, ${ }^{14}$ já citado na RS de Huisstede e seus colaboradores, de $2010 .{ }^{16}$

\section{Resumo crítico dos resultados obtidos}

A eficácia dos corticoides orais a curto e médio prazo no alívio sintomático da STC é evidenciada por vários ECA metodologicamente bem concebidos. Na maioria dos estudos, a administração de $20 \mathrm{mg}$ de prednisolona por duas semanas, com esquema de desmame progressivo, conduz ao alívio temporário dos 
TABELA 3. Guidelines incluídas na revisão e suas características

\begin{tabular}{l|l} 
Guideline & \multicolumn{1}{c}{ Recomendações } \\
\hline $\begin{array}{l}\text { American Academy of Orthopaedic } \\
\text { Surgeons }(\mathbf{2 0 1 6})^{17}\end{array}$ & $\begin{array}{l}\text { De acordo com esta guideline, existe evidência moderada de que os corticoides orais } \\
\text { melhoram os outcomes dos pacientes com síndroma do túnel cárpico em comparação } \\
\text { com o placebo. }\end{array}$
\end{tabular}

sintomas. Este alívio parece ser menos eficaz e duradouro comparativamente à administração local de corticoides, como evidenciado no ECA incluído nesta RBE. ${ }^{5}$ No entanto, os autores referem como limitação do ECA incluído nesta revisão o facto de ter sido usado um corticoide que não está disponível na sua forma oral em Portugal e na Europa.

Outros estudos e revisões sistemáticas apontam uma melhoria significativa nos sintomas às quatro semanas, oito semanas, três meses ou doze meses, ${ }^{2-14}$ não sendo consequentemente possível apontar com certeza a duração média da melhoria clinicamente significativa induzida pela corticoterapia oral; no entanto, os autores desta RBE concluem ser provável que este efeito traduzido pela melhoria sintomática e funcional se mantenha durante pelo menos quatro semanas.

A opção da corticoterapia infiltrada não é geralmente exequível nos cuidados de saúde primários, não sendo considerada no leque de opções do médico de família numa primeira abordagem à STC. A eficácia da corticoterapia oral é documentada de forma robusta por vários estudos, graças à realização de uma avaliação clínica pré e pós intervenção e também, em alguns deles, no decurso da mesma, assim como avaliações eletrofisiológicas, fundamentando as melhorias clínicas numa efetiva modificação neuro-funcional do nervo mediano. No entanto, esta melhoria é apenas documentada de forma sustentada para um tipo de corticoide oral: a prednisolona. O ECA incluído nesta revisão aponta para uma melhoria clínica significativa encontrada também com outro corticoide oral: a triamcinolona. Tendo isto em conta, é possível que a melhoria dos outcomes na STC possa ser um efeito de classe dos corticoides orais e não exclusivo da prednisolona. Mais ensaios clínicos, incluindo outras formulações orais de corticoides, são necessários para concluir que este se trata de um efeito benéfico da classe farmacológica. Por outro lado, os autores destacam que a triamcinolona não está dispo- nível em Portugal nem na Europa na sua formulação oral, o que constitui uma limitação na aplicabilidade das conclusões deste estudo.

Outra limitação encontrada é o facto de a generalidade das amostras dos estudos incluídos ser relativamente reduzida e o tempo de follow-up na sua maioria curto, o que dificulta a generalização das conclusões e achados. Por outro lado, não existem ECA realizados com amostras extraídas de populações seguidas nos cuidados de saúde primários, sendo provável que as populações estudadas apresentem quadros sintomáticos e funcionais mais graves comparativamente à tipologia da população dos cuidados de saúde primários.

Nenhum estudo analisou a eficácia da corticoterapia em associação com outras modalidades terapêuticas, nem estratificou a sua eficácia de acordo com a gravidade dos sintomas iniciais, o que dificulta a seleção dos pacientes que mais poderão beneficiar desta terapêutica. Também não foi avaliada a eficácia do retratamento com corticoterapia oral após recidiva sintomática em nenhum dos estudos contemplados na presente RBE ou nas RS incluídas, ou abordada essa opção terapêutica.

Uma das limitações encontradas radica no facto de nenhum estudo ter comparado a eficácia dos corticoides orais com os fármacos gabapentinoides, frequentemente usados no controlo sintomático do STC, pese embora estes últimos não apresentarem elevado nível de evidência quanto à sua eficácia isolada no tratamento do STC, conforme concluiu uma recente RBE realizada por Neves e colaboradores. ${ }^{18}$

Outra das limitações identificadas reside no facto de que em nenhum dos estudos é feita uma definição temporal exata de curto, médio e longo prazo. De facto, através da análise dos resultados e das conclusões dos diversos estudos depreendem-se definições temporais diferentes entre os mesmos. Através da interpretação 
dos resultados e das conclusões da maior parte dos estudos, os autores desta revisão consideraram até oito semanas como curto prazo, entre oito e doze semanas como médio prazo e superior a doze semanas como longo prazo.

Assim, a evidência relativamente à eficácia da corticoterapia oral no alívio sintomático a curto prazo da STC (até oito semanas) é elevada; no entanto, a evidência é menor no que respeita à sua eficácia a médio e longo prazo, sendo neste caso de nível moderado.

\section{CONCLUSÕES}

Concluindo, os autores consideram que a corticoterapia oral pode ser usada no alívio temporário dos sintomas da STC a curto prazo, quer sensitivos quer motores num regime de 20mg diários de prednisolona durante duas semanas, com eventual esquema de desmame, quer como primeira linha quer como alternativa após falência ou efeitos secundários de outros tratamentos, nomeadamente com gabapentinoides (SORT A). O seu uso no tratamento a médio e a longo prazo dos sintomas apresenta um nível de evidência mais baixo, podendo ser considerado nos casos refratários a outras terapias (SORT B). Mais estudos são recomendados, nomeadamente no âmbito dos cuidados de saúde primários.

\section{AGRADECIMENTOS}

Os autores desejam agradecer aos médicos especialistas de medicina geral e familiar Dr. Hélder Sousa, Dra. Margarida Brandão e Dr. Rui Rocha, pela revisão e orientação dada na realização desta revisão.

\section{REFERÊNCIAS BIBLIOGRÁFICAS}

1. Werner RA, Andary M. Carpal tunnel syndrome: pathophysiology and clinical neurophysiology. Clin Neurophysiol. 2002;113(9):1373-81.

2. Huisstede BM, Randsdorp MS, van den Brink J, Franke TP, Koes BW, Hoogvliet P. Effectiveness of oral pain medication and corticosteroid injections for carpal tunnel syndrome: a systematic review. Arch Phys Med Rehabil. 2018;99(8):1609-22.e10.

3. Shiri R, Miranda H, Heliövaara M, Viikari-Juntura E. Physical work load factors and carpal tunnel syndrome: a population-based study. Occup Environ Med. 2009;66(6):368-73.

4. Becker J, Nora DB, Gomes I, Stringari FF, Seitensus R, Panosso JS, et al. An evaluation of gender, obesity, age and diabetes mellitus as risk factors for carpal tunnel syndrome. Clin Neurophysiol. 2002;113(9): 1429-34.

5. Dewi JI, Sadeli HA, Kurniani N, Gunadharma S. A randomized study camparing oral versus injection triamcinolone in carpal tunnel syndrome. Neurology Asia. 2009;14(2):121-7.
6. Ono S, Clapham PJ, Chung KC. Optimal management of carpal tunnel syndrome. Int J Gen Med. 2010;3:255-61.

7. Hamamoto Filho PT, Leite FV, Ruiz T, Resende LA. A systematic review of anti-inflammatories for mild to moderate carpal tunnel syndrome. J Clin Neuromuscul Dis. 2009;11(1):22-30.

8. Chang MH, Ger LP, Hsieh PF, Huang SY. A randomised clinical trial of oral steroids in the treatment of carpal tunnel syndrome: a long term follow up. J Neurol Neurosurg Psychiatry. 2002;73(6):710-4.

9. Wong SM, Hui AC, Tang A, Ho PC, Hung LK, Wong KS, et al. Local vs systemic corticosteroids in the treatment of carpal tunnel syndrome. Neurology. 2001;56(11):1565-7.

10. Hui AC, Wong SM, Tang A, Mok V, Hung LK, Wong KS. Long-term outcome of carpal tunnel syndrome after conservative treatment. Int J Clin Pract. 2004;58(4):337-9.

11. Page MJ, Massy-Westropp N, O'Connor D, Pitt V. Splinting for carpal tunnel syndrome. Cochrane Database Syst Rev. 2012;2012(7): CD010003.

12. Mishra S, Prabhakar S, Lal V, Modi M, Das CP, Khurana D. Efficacy of splinting and oral steroids in the treatment of carpal tunnel syndrome: a prospective randomized clinical and electrophysiological study. Neurol India. 2006;54(3):286-90.

13. O'Connor D, Marshall S, Massy-Westropp N. Non-surgical treatment (other than steroid injection) for carpal tunnel syndrome. Cochrane Database Syst Rev. 2003;2003(1):CD003219.

14. Chang MH, Chiang HT, Lee SS, Ger LP, Lo YK. Oral drug of choice in carpal tunnel syndrome. Neurology. 1998;51(2):390-3.

15. Marshall S, Tardif G, Ashworth N. Local corticosteroid injection for carpal tunnel syndrome. Cochrane Database Syst Rev. 2007;(2):CD001554.

16. Huisstede BM, Hoogvliet P, Randsdorp MS, Glerum S, van Middelkoop M, Koes BW. Carpal tunnel syndrome. Part I: effectiveness of nonsurgical treatments - a systematic review. Arch Phys Med Rehabil. 2010; 91(7):981-1004.

17. American Academy of Orthopaedic Surgeons. Management of carpal tunnel syndrome evidence-based clinical practice guideline [Internet]. AAOS; 2016.Available from: https://aaos.org/globalassets/quality-andpractice-resources/carpal-tunnel/management-of-carpal-tunnel-syndrome-7-31-19.pdf

18. Neves CM, Vasconcelos A, Carneiro M, Fevereiro M. Uso da gabapentina na melhoria sintomática da síndrome do túnel cárpico: qual a evidência? Rev ADSO. 2018;6(8):23-8.

19. Furlan AD, PennickV, Bombardier C, van Tulder M. 2009 Updated method guidelines for systematic reviews in the Cochrane Back Review Group. Spine. 2009;34(18):1929-41.

\section{CONFLITO DE INTERESSES}

Os autores declaram não possuir quaisquer conflitos de interesse.

\section{ENDEREÇO PARA CORRESPONDÊNCIA}

Nuno Teles Pinto

E-mail: Dr.nunotelespinto@gmail.com

https://orcid.org/0000-0002-1524-9218

Recebido em 22-11-2019

Aceite para publicação em 01-07-2020 


\section{ABSTRACT}

\section{EFFICACY OF ORAL CORTICOIDS IN RELIEVING SYMPTOMS OF CARPAL TUNNEL SYNDROME: WHAT IS THE CURRENT EVIDENCE?}

Introduction: Carpal tunnel syndrome (CTS) is caused by compression of the median nerve as it travels through the carpal tunnel and is the most common upper limb neuropathy.

Objective: To review and analyze the current evidence on the influence of oral corticosteroids on the diagnosis of patients with carpal tunnel syndrome.

Material and Methods: Research for guidelines (GL), randomized controlled clinical trials (RCT), systematic reviews (SR) and meta-analyzes (MA) published between 2009 and 2019, in multiple databases and evidence medical sites from PubMed, The Cochrane Library, DARE, Bandolier, TRIP Database, BMJ Evidence-Based Medicine, National Guideline Clearinghouse, NICE, Canadian Medical Association Practice Guidelines Infobase e Primary Care Clinical Practice Guidelines, using the MeSH terms: "carpal tunnel syndrome" and "corticosteroids". It was applied the SORT scale of the American Academy of Family Physicians for assigning levels of evidence (LE) and strength of recommendations (SR).

Results: Of the 172 articles retrieved by the research, six were selected: one RCT, four SR and one GL. The effectiveness of corticosteroids in the short- medium term on symptoms control of CTS is evidenced by several methodologically well designed RCTs and SR. In most studies, oral administration of prednisolone $20 \mathrm{mg}$ for two weeks led to temporary symptom relief.

Conclusions: Oral corticosteroids can be used in the temporary treatment of short-term CTS symptoms using prednisolone 20 $\mathrm{mg}$ daily for two weeks (SORT A). Its use in the medium- and long-term treatment has a lower level of evidence (SORT B).

Keywords: "carpal tunnel syndrome"; "corticosteroids". 